\title{
Vaccination Hesitancy and Conspiracy Beliefs in the UK During the SARS-COV-2 (COVID-19) Pandemic
}

\author{
Alison M. Bacon ${ }^{1}(\mathbb{D})$. Steven Taylor ${ }^{2}$ \\ Accepted: 21 September 2021 / Published online: 1 October 2021 \\ (c) International Society of Behavioral Medicine 2021
}

\begin{abstract}
Background Conspiracy beliefs about vaccination along with vaccination hesitancy are threats to achieving population immunity during the SARS-COV-2 (COVID-19) pandemic. This longitudinal study aimed to clarify the association between these and non-monetary incentives to vaccination in the UK.

Method Data were collected at three points: (1) before and (2) after the development of a vaccine and (3) after the vaccination programme was underway. At Time 1, participants completed measures of general and COVID-19-specific concerns about vaccination and belief in conspiracy theories. At times 2 and 3, participants reported their intentions whether or not to have the SARS-CoV-2 vaccine. Those who were hesitant provided qualitative comments about what might change their decision. Results Vaccination hesitancy decreased between times $1(54 \%)$ and $3(13 \%)$. There were small effects of conspiracy beliefs on vaccine hesitancy, but only at time 1 . Most concerns and reported incentives were related to safety, although at time 2, incentives included endorsement by trusted public figures. By time 3 , only a minority of participants $(N=18)$ were adamantly against vaccination, stating that nothing would change their minds.

Conclusion Vaccination hesitancy declined in the UK during the course of the study. However, concerns about vaccine safety remained and could jeopardise the vaccination programme should any adverse effects be reported. Conspiracy beliefs seem to play only a minor role in hesitancy and may continue to decrease in importance with a successful vaccination programme. Understanding motivations behind vaccination hesitancy is vital if we are to achieve population immunity.
\end{abstract}

Keywords COVID-19 $\cdot$ Vaccination · Vaccination hesitancy $\cdot$ Conspiracy theories $\cdot$ SARS-COV-2 $\cdot$ Pandemic

\section{Introduction}

In November 2020, the development was announced of a potentially effective vaccine against SARS-COV-2, the novel coronavirus causing COVID-19. While welcome news, for a vaccination programme to achieve herd immunity, 65-70\% of a population must be immunized [1]. A major barrier to achieving this goal is the prevalence of vaccination hesitancy, which is reluctance or refusal to be vaccinated. This is an annual problem for the prevention of seasonal influenza [1] and has presented challenges in previous pandemics, such as the $2009 \mathrm{H} 1 \mathrm{~N} 1$ outbreak [2, 3]. Identifying the

Alison M. Bacon

ambacon@plymouth.ac.uk

1 School of Psychology, University of Plymouth, Plymouth, UK

2 Department of Psychiatry, The University of British Columbia, Vancouver, BC, Canada prevalence and reasons for vaccination hesitancy, and how people might be best encouraged to accept vaccination, is imperative.

In the context of SARS-COV-2 vaccination, reported hesitancy rates in 2020 (prior to the availability of a vaccine) were $14 \%$ in the UK, and belief that the virus was artificially created was strongly associated with hesitancy [4]. In another 2020 pre-vaccine survey [5], 20\% of Canadian and $25 \%$ of American respondents said they would not get vaccinated. Hesitancy was correlated with beliefs that vaccine production had been rushed, that the risks of vaccination outweighed the benefits and that vaccines were a ploy by large pharmaceutical companies to increase their profits [5]. This latter issue is linked to a conspiracy theory that SARS-COV-2 was created in order for the pharmaceutical industry to create a highly profitable vaccine [6]. The strongest incentive for seeking vaccination against SARSCOV-2 was reassurance that the vaccine is safe and thoroughly tested [5]. These results are of interest as beliefs in 
the disease being artificial and that vaccines are unsafe are key factors in medical conspiracy theories [3,7]. The present study presents a three-wave longitudinal investigation into vaccination intention in the $\mathrm{UK}$, with data collected before and after a vaccine was developed, and again once the vaccination programme had begun.

Conspiracy theories are attempts to explain the causes of significant events in terms of secret plots by powerful people or organisations [7]. These theories arise out of uncertainty and a perception of personal threat; hence, pandemics are fertile grounds for the proliferation of conspiracy theories, which may exacerbate vaccination hesitancy [3]. Conspiracy theories are spread on traditional and social media and are difficult to disprove due to the very secrecy of the forces supposedly at work [8-11]. There are individual differences in the tendency to believe in conspiracy theories, and belief in one conspiracy theory tends to be associated with belief in others [3]. However, different forms of conspiracies have distinct implications [12,13] with risk assessment and subsequent behaviour related to the extent by which they downplay or deny the threat. The conspiracy theories most relevant to the pandemic suggest that powerful groups have manipulated the situation to advance their own agenda, for instance, that the virus was created to reduce the population or that the situation has been exaggerated in order to control people through the imposition of behavioural rules, or by implantation microchips during vaccination. Conversely, some theories suggest that the threat has been downplayed, to protect the economy or to avoid panic [13]. One study found that nearly half of 1700 participants surveyed in the UK, USA and Australia believed that authorities are hiding the truth about SARS-COV-2 [13]. Importantly in the present context, conspiracy beliefs have been associated with unsubstantiated fears of vaccination and unwillingness to get vaccinated, as well as non-compliance with government safety guidelines, such as mask wearing [13-16].

In the present study, we examined vaccination intention over three time points: before and after the announcement that a vaccine had been developed, and again once the UK vaccination programme was underway, thus allowing us to identify whether intentions changed with the imminent opportunity for vaccination. At time 1, 894,690 people had tested positive for SARS-COV-2 in the UK, and 58,164 deaths were associated to the virus. By time 3 , the UK morbidity rate had risen to almost 3.5 million and mortality to over 90,000 cases [17]. Given that vaccination hesitancy may arise from, or be influenced by, fears about vaccine safety [5], we expected most hesitancy (and therefore lower intention rates) at time 1 when there was no information about potential vaccines and pandemic concerns were generally reported to be high. By time 3 , when the vaccination programme was underway and no ill effects had been reported, we expected to observe lower hesitancy rates, and hence higher levels of intention to be vaccinated, because many people will have been reassured about their safety concerns. We also expected that strong conspiracy beliefs would moderate the relationship between concern and intention, such that intention would be higher when levels of conspiracy belief were low. Specifically, we predicted that

1. Vaccination hesitancy would decrease from Time 1 to Time 3

2. Vaccination hesitancy would be associated with vaccinerelated concerns (both in general and when specifically related to SARS-COV-2), and to belief in conspiracy theories

3. Belief in conspiracy theories would account for variance in vaccination hesitancy beyond that accounted for by concerns about vaccination and would moderate the relationship between concerns and hesitancy

4. The most strongly endorsed incentives to vaccination would be those which address concerns about safety

\section{Methods}

\section{Participants}

A power analysis using G-Power software [18] indicated a sample size of 208 was required for a medium effect size in the planned regression analysis [19]. Participants $(N=212)$ were recruited from www.prolific.ac.uk, an online research recruitment platform, and agreed to participate. They were each paid $£ 2.50$. Socio-economic status (SES) was assessed by the MacArthur Ladder Scale, which ranks self-reported social class on a ladder with 10 rungs [20] — the higher rungs represent individuals who have more money, education and prestigious jobs. The mean report was 5.35 ( $S D=1.61$, range $1-9)$, with 52 people $(25.5 \%)$ placing themselves on the top three rungs and 28 (9\%) on the bottom three rungs. Most (87.7\%) identified as White, 5 (2.5\%) as Black, $13(6.1 \%)$ as Asian and $7(3.3 \%)$ as mixed race/ other. One participant did not disclose ethnicity. Participants reported home addresses throughout all regions of the UK. At times 2 and 3, the same participants were approached via Prolific.ac.uk. Participation was voluntary and those that took part were paid $£ 2$. Table 1 presents participant demographic data at all three time points.

\section{Materials and Procedures}

The study was approved by the first author's university faculty ethics committee and conducted online. At each of the three stages, participants were required to read details of the study and ethical issues and check a box to give consent before the study could begin. 
Table 1 Demographic characteristics and vaccination intentions by time

\begin{tabular}{|c|c|c|c|}
\hline & Time $1(N=212)$ & Time $2(N=155)$ & $\begin{array}{l}\text { Time } 3 \\
(N=147)\end{array}$ \\
\hline Males & $87(41 \%)$ & $73(47 \%)$ & $64(44 \%)$ \\
\hline Females & $125(59 \%)$ & $82(53 \%)$ & $83(56 \%)$ \\
\hline White & $186(88 \%)$ & $137(88 \%)$ & $131(89 \%)$ \\
\hline Mean Age (SD) & $33.2(11.8)$ & $33.7(11.5)$ & $33.5(11.7)$ \\
\hline Mean SES (SD) & $5.3(1.6)$ & $5.3(1.6)$ & $5.3(1.6)$ \\
\hline \multicolumn{4}{|c|}{ Vaccination intention: number $(\%) *$} \\
\hline Yes & $158(75 \%)$ & $103(66 \%)$ & $119(81 \%)$ \\
\hline No & $54(25 \%)$ & $41(27 \%)$ & $19(13 \%)$ \\
\hline Undecided & - & $11(6 \%)$ & $9(6 \%)$ \\
\hline
\end{tabular}

*At times 2 and 3, yes reflects total responses at points 4 (likely) or 5 (extremely likely) when asked their intentions. Those who responded at scale point 1 (extremely unlikely) or 2 (unlikely) are classed as No, and those who responded at point 3 are counted as undecided

Time 1: Data were collected between 16 and 19 October 2020. Participants completed the following measures:

Vaccination Attitudes Examination Scale [21] We presented two versions of this scale, the first in its original form as a measure of attitudes to vaccination in general and then in revised form whereby the items were slightly reworded so that they reflected attitudes towards COVID-19 vaccinations specifically, for instance, I feel safe after being vaccinated became I will feel safe after being vaccinated against COVID-19. The two scales were presented in this order, but within each scale, the 12 items were presented in different randomised order for each participant. Both versions contain four subscales, all of which showed good reliability with the present sample: mistrust of vaccine benefit (general $\alpha=0.92$, COVID $\alpha=0.75$ ), concerns about unforeseen future effects (general $\alpha=0.80$, COVID $\alpha=0.80$ ), concerns about commercial profiteering by pharmaceutical companies (general $\alpha=0.86$, COVID $\alpha=0.79$ ) and preference for natural immunity (general $\alpha=0.90, \operatorname{COVID} \alpha=0.84$ ).

Participants were also asked a single forced choice yes/ no question to measure vaccination intention: "If a vaccine for COVID-19 was available, would you get vaccinated?" A "don't know" or "uncertain" response option was omitted because it defers endorsing a decision. Participants who answered "No" to this question, were then presented with the list of 21 possible incentives used in previous research [5]. They were asked to rate whether each incentive would increase the likelihood of their getting vaccinated on a 5 -point scale where $0=$ definitely would not and $4=$ definitely would.

The Conspiracy Mentality Questionnaire (CMQ) [7] is a 5 -item scale which measures the general tendency to believe in conspiracy theories such as Events which superficially seem to lack a connection are often the result of secret activities. Participants indicate the extent to which they believe each statement to be true on a scale where $0=$ Certainly not and $10=$ Certain. The scale showed good reliability with the present sample $(\alpha=0.86)$.

Time 2: Development of a vaccine was announced on 9 November 2020 and time 2 data were collected between 17 and 19 November. We contacted all time 1 respondents as follows:

You recently completed a survey for us where we asked for your thoughts about the prospect of a vaccination for COVID-19. Since then, the government has announced the development of a vaccine which is said to be effective against the virus. They have secured supplies of the vaccine with a view to beginning a vaccination programme as soon as possible. In the light of this new information, please indicate how likely you are to have this vaccine if it is offered to you within the next 3 months.

A 5-point response scale was presented whereby $1=$ extremely unlikely, $2=$ unlikely, $3=$ neutral, $4=$ likely and $5=$ extremely likely.

We received 156 responses at time 2 (73\% of the original sample). One was disregarded as the participant ID number did not match any of those from the main study (presumably a typographic error by the respondent), hence at time $2, N=155$. The quick turnaround for study 2 was achievable because we used Prolific.ac.uk as our online recruiting resource. Although responses are anonymous, each person does have an ID code, and it is possible to contact respondents using that. The platform also attracts a rapid response to surveys generally.

Time 3: The UK vaccination programme began on 8 December 2020, and time 3 data were collected between 14 and 15 January 2021 when over two million people in the UK had been vaccinated [22]. Procedures replicated those for time 2 except that our instructions stated that the vaccination programme was already underway, rather than imminent. We received 147 responses, $69 \%$ of the initial sample, and $95 \%$ of the sample at time 2 .

\section{Analysis}

Analysis was conducted using IBM SPSS v. 25. Differences in vaccination intention levels across the time points were compared using chi-square tests for differences in proportions [23]. Relationships between variables were examined using two-tailed Pearson's product-moment correlations. Responses on the five CMQ items were significantly intercorrelated, and all refer to nefarious activities of secret/ powerful others, rather than to specific conspiracy theories, or their explanations for the SARS-COV-2 pandemic. As 
such, we computed the mean score across all five items for use in further analysis. This approach reduced the potential for multicollinearity in regression and also the number of variables, including which was desirable given our lower sample sizes at times 2 and 3. Regression analyses were conducted to examine the extent to which concerns about vaccinations and conspiracy beliefs accounted for variance in vaccination intention. At time 1, intention data were dichotomous $(\mathrm{No}=0$; Yes $=1)$, and so, the analysis conducted was binomial. At times 2 and 3, the data reflects responses on a 5 -point scale from $1=$ extremely unlikely to be vaccinated to $5=$ extremely likely to be vaccinated, so regular linear regression was used. We adopted an intentionto-treat approach at times 2 and 3 such that baseline data were included in the analysis for participants who took part at time 1 but dropped out subsequently. This method avoids possible bias, as attrition may be more frequent in participants with certain characteristics, for instance, particularly poor physical or mental health. This approach also preserves sample size and minimizes type 1 error, as results tend to be conservative [24]. In each regression, we entered age, sex $($ males $=1$, females $=0)$, SES and race $($ white $=1$, other $=0$ ) as covariates, together with scores on the four subfactors of both attitudes to vaccination scales, and the total CMQ score. Moderation analyses were conducted using the PROCESS macro for SPSS v. 3.5, model 1 [25].

\section{Results}

Table 1 presents vaccination intentions at all three timepoints and indicates hesitancy ("No" response) rates at time 1 similar to those reported previously in the USA and
Canada before vaccines became available [5]. Regarding our prediction 1, intention increased, and hesitancy decreased, over time. Chi-square tests for differences in proportions showed that the difference in rates of no responses between times 1 and 2 was not significant, $\chi^{2}(1,211)=0.19, p=0.66$, $95 \% C I[-6.92,11.12]$, though the more substantial decrease between times 2 and 3 was significant, $\chi^{2}(1,154)=9.15$, $p=0.003,95 \% C I[4.98,22.74]$.

Table 2 presents correlations between vaccination intention at all three time points, together with attitudes towards general/COVID-19 vaccines and scores on the CMQ collected at time 1. Correlations indicated a negative association between vaccination intention and negative attitudes towards vaccination at all time points, in line with prediction 2. Associations were moderate to large in magnitude according to established criteria (i.e., 0.30 to 0.50 , or larger). Belief in conspiracy theories was negatively associated with vaccination intention at times 1 and 2 as expected, but no association between these factors was apparent at time 3 . We found no significant associations between conspiracy beliefs and gender, race or age ( $r>0.10$ in every case).

Regression analysis was conducted on data at each time point. At time 1, the binomial regression model accounted for $69 \%$ variance in vaccination intention (Nagelkerke $R^{2}$ ) with $89 \%$ of participants correctly classified. Variance was accounted for by higher levels of mistrust in the COVID vaccines, $\beta=1.53, p<0.001,95 \% C I[2.45,8.73]$ and concern about future effects of COVID vaccines, $B=1.03, p=0.002$, $95 \% C I[1.45,5.37]$. However, contrary to prediction 3 , we observed no significant independent effect of conspiracy beliefs (CMQ score), $p=0.16$. A significant moderating effect of conspiracy belief on the relationship between concerns about future effects of COVID-19 vaccine and
Table 2 Correlations between Covid-19 vaccination hesitancy, negative attitudes towards vaccines and CMQ score

\begin{tabular}{|c|c|c|c|}
\hline & \multicolumn{3}{|c|}{ Vaccination hesitancy } \\
\hline & Time 1 & Time 2 & Time 3 \\
\hline \multicolumn{4}{|l|}{ Negative attitude towards vaccines } \\
\hline \multicolumn{4}{|l|}{ Concerning COVID-19 vaccine } \\
\hline Mistrust of vaccine benefit & $.63 * *$ & $.63 * *$ & $.50 * *$ \\
\hline Worry about unforeseen future negative effects & $.48 * *$ & $.49 * *$ & $.48 * *$ \\
\hline Concern about commercial profiteering & $.59 * *$ & $.54 * *$ & $.44 * *$ \\
\hline Preference for natural immunity & $.51 * *$ & $.43 * *$ & $.31 * *$ \\
\hline \multicolumn{4}{|l|}{ Concerning vaccines in general } \\
\hline Mistrust of vaccine benefit & $.50 * *$ & $.63 * *$ & $.35^{* *}$ \\
\hline Worry about unforeseen future negative effects & $.39 * *$ & $.53 * *$ & $.33 * *$ \\
\hline Concern about commercial profiteering & $.49 * *$ & $.59 * *$ & $.46^{* *}$ \\
\hline Preference for natural immunity & $.46^{* *}$ & $.49 * *$ & $.36^{* *}$ \\
\hline Total CMQ score (conspiracy beliefs) & $.25 * *$ & $.17 *$ & .08 \\
\hline
\end{tabular}

Vaccination intention at time 1 is biserial $(\mathrm{No}=0$, Yes $=1)$. At times 2 and 3, rating scale responses where higher score indicates greater intention to be vaccinated

${ }^{*} p<.05 ; * * p<.01$ 
vaccination intention indicated that intention was lower in the presence of higher levels of conspiracy belief, $\chi^{2}=9.46$, $p=0.002,95 \%$ CI $[-0.46,-0.11]$, as predicted. Note that for this binomial analysis, statistics are reported as log-odds ratios. A Johnson-Neyman analysis showed that the critical value of CMQ score was 3.43 - above this level, the moderation effect is significant. No significant moderating effect on the relationship between mistrust of vaccines and intention was observed, $p=0.67$.

At Time 2, the model accounted for $39 \%$ variance. COVID-specific mistrust, $\beta=0.29, p=0.001,95 \% C I[0.15$, $0.54]$, and apprehension about future effects of the vaccine, $\beta=0.20, p=0.02,95 \% C I[0.04,0.46]$, were still significant factors, together with concerns about profiteering by pharmaceutical companies, $\beta=0.31, p=0.01,95 \% C I[0.08,0.60]$. No significant effects of conspiracy beliefs were observed $(p=0.30)$. No moderating effects of conspiracy beliefs were found. Finally, at time 3, the model accounted for $28 \%$ variance. At this stage, only two factors showed significant effects on intention, concern about future effects of vaccinations in general, $\beta=0.26, p=0.01,95 \% C I 0.09,0.53]$ and about pharmaceutical companies making a profit from COVID-19 vaccine, $\beta=0.37, p=0.01,95 \% C I[0.11,0.65]$. No moderating effects of conspiracy belief were observed.
For participants who said they did not intend to get vaccinated at time 1 , we computed the proportion who stated they probably or definitely would be vaccinated if offered each of 21 incentives (Table 3). The most persuasive incentives were those which suggested the vaccine to be safe and effective as expected (prediction 4). The least effective incentives at time 1 were those pertaining to promotion of the vaccine through the media, religious or political leaders. The table also presents data from participants who stated a negative intention at time 1 and then continued to state that they were unlikely or extremely unlikely to be vaccinated at times 2 and 3 . What is notable at time 2 (development of vaccine recently announced) is an increase in the importance of incentives concerning vaccine endorsement by trusted sources and the media. However, by time 3 (vaccination underway), these incentives became less important, and those related to safety and testing were again most favoured.

We compared responses across the three timepoints to estimate the rate of change in likelihood of vaccination. Of the 54 participants who stated they would not get vaccinated at Time 1, $39(72 \%)$ remained in the study across all three stages. By Time 3, just under a third of these participants (30\%) were still stating that they were unlikely or extremely unlikely to have the vaccine. A further four remained

Table 3 Incentives to get vaccinated endorsed at time 1

\begin{tabular}{|c|c|c|c|}
\hline \multirow[b]{2}{*}{ Incentive } & \multicolumn{3}{|c|}{$\%$ endorsement* } \\
\hline & $\begin{array}{l}\text { Time } 1 \\
(N=54)\end{array}$ & $\begin{array}{l}\text { Time } 2 \\
(N=43)\end{array}$ & $\begin{array}{l}\text { Time } 3 \\
(N=13)\end{array}$ \\
\hline If I was convinced that the vaccine had been rigorously tested & 48 & 42 & 31 \\
\hline If I saw that enough people were safely vaccinated without negative side effects & 48 & 44 & 30 \\
\hline If I saw that enough people who got the vaccine didn't get sick with COVID-19 & 48 & 38 & 62 \\
\hline If I saw that my friends and family didn't have negative side effects from the vaccine & 46 & 37 & 23 \\
\hline If getting vaccinated was a requirement for my job & 41 & 40 & 23 \\
\hline If I thought the health authorities were trustworthy & 39 & 33 & 31 \\
\hline If I was convinced that getting vaccinated helped protect vulnerable members of my community & 37 & 42 & 38 \\
\hline If getting vaccinated was required by my government & 35 & 26 & 0 \\
\hline If a trusted health care worker told me to get vaccinated & 31 & 23 & 7 \\
\hline If I knew that I was not being exploited by the pharmaceutical industry & 26 & 25 & 8 \\
\hline If getting vaccinated was required for me to attend social or sporting events & 26 & 28 & 15 \\
\hline If someone I knew died from COVID-19 & 24 & 14 & 0 \\
\hline I received a financial incentive & 20 & 30 & 30 \\
\hline If I was assured that the government wasn't controlling the vaccine & 17 & 35 & 30 \\
\hline If someone I knew got sick with COVID-19 & 17 & 9 & 0 \\
\hline If someone I knew was hospitalized because of COVID-19 & 17 & 12 & 0 \\
\hline If I received some other incentive (e.g., discount coupon) & 9 & 14 & 7 \\
\hline If a news source that I trust promoted vaccination & 6 & 40 & 0 \\
\hline If religious leaders in my community said I should get vaccinated & 6 & 18 & 0 \\
\hline If the Prime Minister promoted the vaccine & 4 & 16 & 0 \\
\hline If vaccination was promoted in my social media network & 2 & 18 & 0 \\
\hline
\end{tabular}

$* \%$ of endorsement at point 3 on the response scale (probably would) and point 4 (definitely would) 
undecided. Conversely, of the 158 participants who stated they intended to be vaccinated at Time 1, just $6(4 \%)$ had changed their mind by Time 3 and said they were unlikely or extremely unlikely to get vaccinated. A further 5 were undecided. Although indicative that changing circumstances may alter behaviour in both directions, these numbers are too low to draw any firm conclusions.

\section{Discussion}

This study aimed to examine the extent of vaccination hesitancy in the UK and some of the reasons for this. We collected data over three waves; before the prospect of a vaccine, when a vaccine had been approved for use, and when the UK vaccination programme had been underway for a few weeks. Our data suggest that while most people are happy to be vaccinated against COVID-19, a significant proportion remain unconvinced with many concerns about safety in particular. Vaccination hesitancy rates decreased across the time points as expected (prediction 1), with the greatest increase between times 2 and 3. Perception of risk is associated with uncertainty and unusual, and unexpected events are perceived as particularly frightening [26]. At time 1 , government and media reports suggested this was a new disease, and there was no imminent prospect of a vaccine as research was starting from scratch [27]. We suggest that public confidence increased as vaccines were rolled out with no reports of adverse effects. Although there have been some cases of thrombotic conditions associated with one of the SARS-COV-19 vaccines administered in the UK, these had not been reported at the time data was collected.

Vaccination hesitancy was associated significantly with conspiracy belief in line with prediction 2 , but only at times 1 and 2. Our regression analyses did not indicate that conspiracy beliefs independently accounted for variance in intention, as we had expected (prediction 3). However, the predicted moderating effect of beliefs on the association between vaccine-related concerns and intention was observed, though only at time 1 , and only for the effect of worries specifically about as yet unknown future effects of Covid-19 vaccination. These concerns were more likely to reduce vaccination intention (and by implication increase vaccination hesitancy) when conspiracy belief was high. At times 1 and 2, a lack of trust in COVID-19 vaccination, together with worries about possible future side effects, explained significant variance in intention to vaccinate. By time 3, the only significant factors in explaining hesitancy were concerns about future effects of vaccines in general (not necessarily COVID related), and about profiteering by pharmaceutical companies. This concern has previously been linked to conspiracy theories suggesting that SARSCOV-2 was manufactured in order for the pharmaceutical industry to create a highly profitable vaccine [6]; however, we observed no effects of conspiracy beliefs at Time 3 .

In line with previous research [5], we expected that the incentives to vaccination most strongly endorsed by hesitant participants would be those concerned with reassurances of safety (prediction 4). This was indeed the case at time 1 and was endorsed by qualitative data collected at times 2 and 3 . Sixty-six per cent of respondents at time 2 (after the development of vaccines had been announced) reported a belief that development had been rushed, and/or vaccines cannot have been thoroughly tested. It is also notable at time 2 that a good proportion of participants who were still hesitant had, at time 1, favoured incentives concerning endorsement of the vaccine in the media and by trusted figures. By time 3, when only $13 \%$ of participants reported hesitancy, most people seemed to have changed their mind as those incentives were not endorsed at all. At this point, most qualitative comments reflected a firm resistance to vaccination altogether or other factors such as a wish to have the vaccine by nasal spray rather than injection.

These results present some potentially important insights for health communication.

Firstly, at time 2, we observed the potential importance of incentives linked to pro-vaccination endorsement by public figures. Although such incentives were endorsed at time 1 , they reflected only a small minority of participants and were only observed as predominant incentives at time 2 , soon after the development of an effective vaccine had been announced, but before its rollout. This may present a window of opportunity for bringing well-known dignitaries or celebrities into health communication. Vaccination intention is related to the perception of sufficient information to make an informed decision. Secondly, hesitancy rates fell over time, decreasing in line with increased availability of vaccination. Vaccination attitudes may similarly change over time with effective pro-vaccination public education [5]. We did not measure that factor specifically, but it may be that an effective vaccination programme will, over time, become its own favourable publicity. Decreasing hesitancy may be a trend which perpetuates as long as no detrimental effects of the vaccine become apparent. However, favoured incentives at time 3 predominantly return to reassurances of safety; therefore, any negative publicity may have a highly detrimental effect now that vaccination is taking place. Presentation of pro-vaccination information, especially about safety, by trusted public figures may potentially allay public concern and uncertainty. However, the choice of ambassadors and the timing of messaging must be carefully considered. Gaining public trust will be key [28]. There is evidence of low public trust in politicians and policy-makers in both present and previous pandemic [3, 5], and celebrity endorsement may not appeal to all demographics. Messages must achieve three key goals: (1) convince people that vaccines 
are thoroughly tested and safe; (2) demonstrate the necessity of vaccination, that people are at risk and the vaccination can ameliorate that risk; and (3) gain public trust. They must be seen to be endorsed not only by politicians, but also by others who the public perceive as being in touch with "real" life.

Finally, some time 3 respondents were still resistant to vaccination. Most gave no explanation for their attitudes, simply responding "nothing" to the qualitative question asking what might change their mind. Worryingly, some participants still seem convinced that they are not at risk. Numbers are small to draw definitive conclusions, and research can usefully examine these issues further to ensure that communications are geared to appeal to the appropriate demographic.

Perhaps surprisingly given evidence from previous disease outbreaks, we found relatively little role for conspiratorial thinking, at least not once vaccines had been developed. Conspiracy theories around the nature and origin of SARSCOV-19 proliferate on social media, with many articles and videos urging people to ignore scientists and politicians and "do their own research", but we found little effect of such beliefs on vaccination intention after time 1 , a time when there seemed no prospect of an imminent vaccine. Studies in the USA have shown that conspiracy beliefs predict resistance to vaccination [14] and belief in conspiratorial governments is associated with low coherence to social distancing and handwashing in Poland [29]. It may be that conspiracy beliefs are not as widespread in the UK, or not amongst our sample. Most recently, a report in the British Medical Journal has suggested that the accumulating evidence in favour of official pandemic responses such as behavioural restriction, testing and vaccination programmes has openly refuted many conspiracy theories [30]. Furthermore, people with such beliefs tend to be low in trust and perceptions of control, believing in the existence of threatening and subversive plots $[12,13]$. They may therefore not be most likely to volunteer for research studies.

There is evidence that anti-vaccination attitudes may be modified by interventions based on motivational interviewing [9]. This relies on understanding the motivations behind vaccination hesitancy, including belief in conspiracy theories. By working with people's worldviews, as well as their overt attitudes, it may be possible to show how the benefits of vaccination are obscured, and the potential dangers exaggerated, in conspiracies [3]. A further issue not considered in the present research is perceived vulnerability to disease (PVD). PVD can be increased by reading information about the COVID pandemic, including reminders of Government behavioural guidelines, and is found to be associated with support for these lifestyle restrictions [31]. While it may therefore seem expedient to raise anxiety, this may not always translate into the desired behaviours. Although there is considerable evidence that high PVD is associated with conformism, such individuals may also be susceptible to anti-vaccination rhetoric [32], which can thrive in the context of perceived personal vulnerability [13]. In addition, at times 2 and 3, we found respondents who believed themselves not to be at risk of contracting SARS-COV-19. Numbers were too small for detailed investigation, but are sufficient to indicate some prevalence of such beliefs. The relationship between PVD and anti-vaccination attitudes is not fully understood and may present a useful biobehavioural perspective through which to study responses to the pandemic, and how individuals perceive the risks and benefits of vaccination.

A clear strength of this research is that it examined vaccination intention over three time points. It presents new information about how attitudes and intentions may change over time. It has also suggested some ways in which health communications may usefully be targeted. However, there are some limitations, including the use of self-report data, and the recognized problem of attrition in panel studies. Although the recall rate was substantial, the regression analyses were underpowered at times 2 and 3. While we controlled for this statistically to a point by using an intentionto-treat approach, sample size is still a limitation at these timepoints. At time 1, there seemed no prospect of an effective vaccine. However, less than a fortnight later, an imminent vaccination programme was announced. We took advantage of this development to collect longitudinal data, recalling as many time 1 participants as possible. Had we known at the outset how rapidly vaccination development was advancing, we would have planned a longitudinal approach initially and over-recruited at time 1 to account for attrition. Finally, our sample was almost exclusively White. The question of whether vaccination attitudes differ across different ethnic or cultural groups in the UK remains.

In conclusion, vaccination intention and incentive to vaccination appear to be malleable and change as new information and treatments emerge. This is an important finding. It shows that people who are hesitant about vaccination can and do change their minds, and health communication should focus on this issue. Publicity to highlight how medical science is consistently progressing with testing, and the success of vaccination rollout is essential to ameliorate ongoing public concern, and to refute conspiracy theories. Pro-vaccination messages delivered by highly trusted public figures may be helpful. Identification of any particular sectors of society who do not perceive themselves at risk will also be beneficial for targeting health communications involving messengers recognised by that sector of the population. Finally, a minority of people in our studies remained resolutely anti-vaccination throughout, and this was not clearly linked to belief in conspiracies. These "vaccine refusers" also deserve research attention. Understanding the motivations behind vaccination hesitancy is vital if we are to achieve a situation where the population can live alongside the virus in relative safety. 
Data Availability The data associated with this study are available at https://doi.org/10.5281/zenodo.4518697.

\section{Declarations}

Ethics Approval All procedures performed in studies involving human participants were in accordance with the ethical standards of the institutional and/or national research committee and with the 1964 Helsinki declaration and its later amendments or comparable ethical standards.

Informed Consent Informed consent was obtained from all individual participants included in the study.

Competing Interests The authors declare no competing interests.

\section{References}

1. WHO. Ten threats to global health in 2019. 2019. Retrieved 04/02/2021 from https://www.who.int/news-room/spotlight/tenthreats-to-global-health-in-2019.

2. Bangerter A, Krings F, Mouton A, Gilles I, Green EG, Clémance A. Longitudinal investigation of public trust in institutions relative to the 2009 H1N1 pandemic in Switzerland. PLoS One. 2012;7(11):e49806. https://doi.org/10.1371/journal.pone.0049806.

3. Taylor S. The psychology of pandemics: preparing for the next global outbreak of infectious disease. Newcastle upon Tyne, UK: Cambridge Scholars Publishing; 2019.

4. Salali GD, Uysal MS. COVID-19 vaccine hesitancy is associated with beliefs on the origin of the novel coronavirus in the UK and Turkey. Psychol Med. 2020. https://doi.org/10.1017/S0033291720004067.

5. Taylor S, Landry CA, Paluszek MM, Groenewoud R, Rachor GS, Asmundson GJG. A proactive approach for managing COVID-19: the importance of understanding the motivational roots of vaccination hesitancy for SARS-CoV2. Front Psychol. 2020. https://doi.org/10.3389/fpsyg.2020.575950.

6. Lynas M. COVID: top 10 current conspiracy theories. 2020. Retrieved 27/01/2021 from https://allianceforscience.cornell. edu/blog/2020/04/covid-top-10-current-conspiracy-theories/.

7. Bruder M, Haffke P, Neave N, Nouripanah N, Imhoff R. Measuring individual differences in generic beliefs in conspiracy theories across cultures: Conspiracy Mentality Questionnaire. Front Psychol. 2013;4:225. https://doi.org/10.3389/fpsyg.2013.00225.

8. Douglas KM, Sutton RM, Cichocka A. The psychology of conspiracy theories. Curr Dir Psychol Sci. 2017;26:538-42. https:// doi.org/10.1177/0963721417718261.

9. Hornsey MJ, Harris EA, Fielding KS. The psychological roots of anti- vaccination attitudes: a 24 nation investigation. Health Psychol. 2018;37:307-15. https://doi.org/10.1037/hea0000586.

10. Garfin DR, Silver RC, Holman EA. The novel coronavirus (COVID2019) outbreak: amplification of public health consequences by media exposure. Health Psychol. 2020;39:355-7. https://doi.org/ 10.1037/hea0000875355.

11. Mheidly N, Fares J. Leveraging media and health communication strategies to overcome the COVID 19 infodemic. J Public Health Policy. 2020;41:410-20. https://doi.org/10.1057/s41271-020-00247-w.

12. Imhoff R, Lamberty P. A bioweapon or a hoax? The link between distinct conspiracy beliefs about the coronavirus disease (COVID19) outbreak and pandemic behavior. Soc Psychol Personal Sci. 2020;11:1110-8. https://doi.org/10.1177/1948550620934692.

13. Hornsey MJ. Conspiracy theories. In: Jetten J, Reicher SD, Haslam SA, Cruwys T, editors. Together apart: the psychology of COVID-19. London: Sage; 2020. p. 41-6.
14. Romer D, Jamieson KH. Conspiracy theories as barriers to controlling the spread of COVID-19 in the U.S. Soc Sci Med. 2020. https://doi. org/10.1016/j.socscimed.2020.113356.

15. Taylor S, Paluszek M, Landry C, Rachor GS, Asmundson GJG. Worry, avoidance, and coping during the COVID-19 pandemic: a comprehensive network analysis. J Anxiety Disord. 2020;76:102327. https://doi.org/10.1016/j.janxdis.2020.102327.

16. Taylor S, Asmundson GJG. Negative attitudes about facemasks during the COVID-19 pandemic: the dual importance of perceived ineffectiveness and psychological reactance. PLoS One. 2021;16:e0246317. https://doi.org/10.1101/2020.11.17.20233585.

17. UK Government. Coronavirus (COVID-19) in the UK. 2021. Retrieved 20/01/2021 from https://coronavirus.data.gov.uk/.

18. Faul F, Erdfelder E, Buchner A, Lang A-G. Statistical power analyses using $\mathrm{G}^{*}$ Power 3.1: tests for correlation and regression analyses. Behav Res Methods. 2009;41:1149-60. https://doi.org/ 10.3758/BRM.41.4.1149.

19. Cohen J. Statistical power analysis for the behavioral sciences. 2nd ed. Hillsdale, NJ: Erlbaum; 1988.

20. Adler NE, Epel ES, Castellazzo G, Ickovics JR. Relationship of subjective and objective social status with psychological and physiological functioning: preliminary data in healthy, white women. Health Psychol. 2000;19:586-92. https://doi.org/10.1037/0278-6133.19.6.586.

21. Martin LR, Petrie KJ. Understanding the dimensions of antivaccination attitudes: the Vaccination Attitudes Examination (VAX) Scale. Ann Behav Med. 2017;51:652-60. https://doi. org/10.1007/s12160-017-9888-y.

22. NHS. COVID-19 vaccination statistics week ending Sunday 10th January 2021. 2020. Retrieved 16/01/2021 from https://www.england.nhs. uk/statistics/statistical-work-areas/covid-19-vaccinations/

23. Campbell I. Chi-squared and Fisher-Irwin tests of two-bytwo tables with small sample recommendations. Stat Med. 2007;26:3661-75. https://doi.org/10.1002/sim.2832.

24. Gupta SK. Intention-to-treat concept: a review. Perspect Clin Res. 2011;2:109-12. https://doi.org/10.4103/2229-3485.83221.

25. Hayes AF. Introduction to mediation, moderation and conditional process analysis. 2nd ed. New York: Guilford; 2018.

26. Slovic P, Fischhoff B, Lichtenstein S. Cognitive processes and societal risk taking. In: Slovic P, editor. The Perception of Risk. London: Earthscan Publications Ltd; 2000. p. 32-50.

27. Cori L, Bianchi F, Cadum E, Anthonj C. Risk perception and COVID-19. Int J Environ Res Public Health. 2020;17:3114. https://doi.org/10.3390/ijerph17093114.

28. Cairney P, Wellstead A. COVID-19: Effective policymaking depends on trust in experts, politicians, and the public. Policy Des Pract. 2020. https://doi.org/10.1080/25741292.2020.1837466.

29. Oleksy T, Wnuk A, Maison D, Łyś A. Content matters: different predictors and social consequences of general and governmentrelated conspiracy theories on COVID-19. Personal Individ Differ. 2020;168: 110289. https://doi.org/10.1016/j.paid.2020.110289.

30. Oliver D. Covid deniers' precarious Jenga tower is collapsing on contact with reality. BMJ Opinion. 2021. Retrieved 08/02/2021 from https://blogs.bmj.com/bmj/2021/02/01/david-oliver-covid-deniersprecarious-jenga-tower-is-collapsing-on-contact-with-reality/.

31. Bacon AM, Corr PJ. Behavioral immune system responses to coronavirus: a reinforcement sensitivity theory explanation of conformity, warmth towards others and attitudes toward lockdown. Front Psychol. 2020. https://doi.org/10.3389/fpsyg.2020.566237.

32. Murray DR, Schaller M. The behavioral immune system: Implications for social cognition, social interaction, and social influence. Adv Exp Soc Psychol. 2016;53:75-129. https://doi.org/10.1016/bs.aesp.2015. 09.002 .

Publisher's Note Springer Nature remains neutral with regard to jurisdictional claims in published maps and institutional affiliations. 